\title{
Breves reflexões sobre a desconexão entre a pesquisa científica e a inovação tecnológica no Brasil
}

Edmeire Cristina Pereira*

Resumo: Este artigo aborda a desconexão existente no Brasil entre a pesquisa científica e a inovação tecnológica. Evidencia isso por meio do conceito de modelo linear de C\&T que é praticado no país. No entanto, procura mostrar proativamente o papel dos NIT Núcleos de Inovação Tecnológica, criados pela Lei N. 10.973/04, em prol da redução dessa assimetria entre a pesquisa científica e a inovação tecnológica e fortalecimento de uma cultura de proteção dos ativos intangíveis, bem como da transferência de tecnologias pelas ICT - Instituições de Ciência e Tecnologia. Ressalta dois NIT regionais: o NITPAR e a Agência de Inovação UFPR.

Palavras-chave: Inovação tecnológica; C\&T (modelo linear); C\&T (modelo dinâmico de inovação); NIT (Brasil); NITPAR (Paraná); Agência de Inovação UFPR.

Classificação JEL: O32; O34; L32.

\footnotetext{
* Mestre em Biblioteconomia e Ciências da Informação pela Pontifícia Universidade Católica de Campinas (PUC Campinas). Professora Assistente IV do Departamento de Ciência e Gestão da Informação (DECIGI/UFPR) e excoordenadora de propriedade intelectual na Agência de Inovação UFPR. Endereço eletrônico: edmeire@ufpr.br.
} 


\section{Introdução}

Este artigo é um exercício de reflexão acerca da desconexão entre a pesquisa científica produzida pelas ICT - Instituições de Ciência e Tecnologia e a inovação tecnológica produzida pelas empresas brasileiras.

Essa assimetria é um grande desafio para o país, pois segundo Dauscha (2010): "A ciência tem uma oferta, que não foi demandada, ainda, pela indústria". Ou, como diríamos: "procura-se um problema, pois nós temos a solução"...

Como os NIT - Núcleos de Inovação Tecnológica são fundamentais para o fortalecimento do Sistema Nacional de Inovações e foram criados recentemente pela Lei N. 10.973/2004 (Brasil, 2004) serão enfatizados neste artigo. Destacaremos o NIT do Estado do Paraná (NITPAR), criado em 2007 e o NIT da UFPR, denominado de AGITEC (nome fantasia), criado em 2008, pela Resolução COPLAD n. 16/08 (UFPR, 2008). Posteriormente, à sua criação, este NIT teve sua marca (mista) registrada junto ao INPI Instituto Nacional da Propriedade Industrial - www.inpi.gov.br, como Agência de Inovação UFPR.

\section{Modelo linear de C\&T}

Com o lançamento do PAC - Plano de Aceleração do Crescimento www.pac.gov.br, pelo Governo Lula, em meados de novembro de 2007, a C\&T e, por extensão, a inovação e o desenvolvimento sustentável, são temas que, finalmente, parecem ter entrado definitivamente, na agenda nacional, não mais como políticas de governo e sim, como políticas de Estado(PACTI/MCT e PDP/ MDIC). Tal Plano se assenta em prioridades estratégicas e linhas de ação.

Atualmente, no Governo Dilma Rousseff, o trinômio C,T \& I continua a ser prestigiado pelas pastas dos novos ministros empossados no começo de 2011 (e já trocados no início de 2012), para o MCT (Ministério da Ciência e Tecnologia) e o MDIC (Ministério do Desenvolvimento, Indústria e Comércio Exterior).

O MCT, inclusive, incorporou a palavra inovação em sua sigla que, desde de 02/08/11, passou a se chamar Ministério da Ciência, Tecnologia e Inovação (MCTI), durante o lançamento do Plano Brasil Maior. Além desta medida, o Ministro propôs outras, igualmente importantes, tais como: a) assinatura de memorando de intenções entre o seu Ministério e a CNI para criar uma empresa do tipo EMBRAPA, para a indústria, cujo nome será: Empresa Brasileira para Pesquisa e Inovação na Indústria (EMBRAPII); b) fortalecimento financeiro da FINEP, com recursos da ordem de mais $\mathrm{R} \$ 2$ bilhões para a Agência, oriundos do BNDES; c) criação do Programa Ciência Sem Fronteiras, em parceria com o MEC, para oportunizar 75 mil bolsas de estudo para alunos de graduação, mestrado, doutorado ou pós-doutorado e até estágios em empresas estrangeiras, nas melhores universidades do mundo, bem como a vinda de renomados cientistas 
estrangeiros para repartir conhecimentos com cientistas brasileiros; d) tornar o Brasil o primeiro país tropical desenvolvido e avançar rumo à Sociedade do Conhecimento e à Sustentabilidade Ambiental. "Economia do futuro é uma economia do conhecimento", enfatizou o Ministro Mercadante (www.mct.gov. br - acesso em: 12/08/11).

O MDIC, por sua vez, vem trabalhando com a PDP - Política de Desenvolvimento Produtivo, lançada no Governo Lula, em 12/05/08 que, agora, ganhou uma nova "roupagem" em termos de política industrial, com o lançamento do Plano Brasil Maior, em 02/08/11, sendo uma nova política industrial, tecnológica, de serviços e de comércio exterior, pelo período de 2011 2014. Este plano "reduzirá a zero a alíquota de 20\% para o INSS de setores sensíveis ao câmbio e à concorrência internacional e intensivos em mão-deobra, tais como: confecções, calçados, móveis e softwares" (www.mdic.gov.br - acesso em: 12/08/11).

As principais medidas anunciadas foram:

a) o REINTEGRA - Regime Especial de Reintegração de Valores Tributários para as Empresas Exportadoras - que é a devolução ao exportador de bens industrializados $0,5 \%$ da receita da exportação;

b) a regulamentação da Lei de Compras Governamentais (Lei N. 12.349/2010), focada nas áreas de Defesa, Saúde e TICs.;

c) defesa comercial com medidas antidumping;

d) modernização do INMETRO, que passará a se chamar Instituto Nacional de Metrologia, Qualidade e Tecnologia;

e) um novo regime automotivo (veículos acabados e autopeças);

f) PIS-COFINS e desonerações sobre bens de capital;

g) Inovação e financiamento: recursos oriundos do BNDES para a FINEP para ampliação de sua carteira de inovação e outros programas para investimentos, como o Programa de Sustentação de Investimento (PSI), para equipamentos de TICs produzidos no país e o Programa BNDES Revitaliza, incluindo mais um setor, o de autopeças;

h) Conselho Nacional de Desenvolvimento Industrial (CNDI) que terá por função propor ao Presidente da República políticas nacionais e medidas específicas para o desenvolvimento industrial do país e orientações estratégicas gerais para o Plano Brasil Maior. 
No entanto, como afirma Lotufo (2009, p.46 e 49), ainda "persiste uma significativa desconexão entre a pesquisa científica e a inovação tecnológica no Brasil". Para este autor:

\begin{abstract}
“(...) Há necessidade do fortalecimento da política de C\&T\&I em estreita colaboração com a política industrial”. Observa-se um grande hiato entre a produção de conhecimentos científicos, de um lado, e medidos pela produção nacional de artigos científicos e, de outro, a transformação desses conhecimentos em tecnologias e riquezas para o país, medidos pelos depósitos de patentes de invenção".
\end{abstract}

Concordamos parcialmente, com o autor acima, porque a julgar pelas ações governamentais, este player está fazendo a sua parte, haja vista a quantidade de medidas propostas recentemente. Inclusive, de uma maneira histórica e inédita, em reunir os três Ministérios (MCT, MDIC e MEC) para pensar as questões da Inovação para o País. Entretanto, não podemos perder de vista que um país para se tornar realmente desenvolvido, precisa resolver primeiro as suas questões econômicas e sociais, além de passar por alguns estágios de desenvolvimento tecnológico, que não dependem diretamente das pesquisas científicas, pois corroboramos com Mattos e Guimarães (2005, p.5) em seus conceitos de ciência e tecnologia, as suas diferenças, a saber:

\footnotetext{
"Entende-se por ciência o conjunto organizado dos conhecimentos relativos ao universo objetivo, envolvendo seus fenômenos naturais, ambientais e comportamentais. Em geral, a ciência é dita pura ou fundamental, quando desvinculada de objetivos práticos, e aplicada, quando visa conseqüências determinadas". (...)

"Portanto, tecnologia é o conjunto organizado de todos os conhecimentos - científicos, empíricos ou intuitivos - empregados na produção e comercialização de bens e de serviços. (...) A tecnologia é normalmente produzida e levada a sua plena utilização pelo setor produtivo por meio de um sistemático encadeamento de atividades de pesquisa, desenvolvimento experimental e engenharia”. (P\&D\&E)
}

Considerando, então, que, historicamente, no Brasil, essa indissociabilidade entre a ciência e a tecnologia não nos levou à uma independência tecnológica (Nikolsky, 2010), há que se buscar outras alternativas de entendimento e solução para o problema. O Brasil, ainda, investe muito pouco em P\&D. "A meta da PDP é elevar este percentual de $0,65 \%$ em relação ao PIB de 2010, o que requererá um crescimento médio anual de $9,8 \%$ nos gastos privados em P\&D da indústria brasileira" (www.pdp.gov.br - acesso em: 12/08/11). 
Com isso, vem praticando o "modelo linear de C\&T" amplamente criticado pelos estudiosos na literatura, pois entendem que esse modelo pressupõe que as inovações têm de surgir nos laboratórios de P\&D das Universidades e Institutos Tecnológicos, rumar para os mercados e serem transferidos com sucesso para os seus adquirentes de tecnologias, por compras/vendas e/ou licenciamentos. Ora, esse comportamento fica totalmente dependente das competências tecnológicas e econômicas do sistema produtivo e se aplicam muito bem às economias altamente desenvolvidas (Nikolsky, 2010,p.33); o que não é o caso dos países emergentes, tal como o Brasil. Dentro dessa lógica, percebemos que o país se destaca em apenas alguns segmentos muito restritos, por exemplo: petróleo e gás, aviação comercial de médio porte e celulose de fibra curta de eucalipto (Nikolsky, 2010, p.34).

Portanto, há que se ter em mente que a pesquisa tecnológica para existir não carece diretamente do trabalho de seus cientistas e sim, de sua relação com seus usuários, consumidores e os mercados (valor econômico). Nesse sentido, Nikolsky (2010, p.37-38) afirma que:

\begin{abstract}
"Assim, um pais pode inovar e até liderar mundialmente a inovação tecnológica em uma área na qual não tenha domínio científico reconhecido em nível internacional. Tal fato ocorre com os países citados em diversas linhas de produtos e até com o Brasil, que é líder de inovações em nível mundial em aviação a jato regional e não tem expressão significativa em ciências aeronáuticas e dinâmica dos fluidos. Isso é extraordinariamente mais simples e mais rápido do que no modelo anterior, pois pode-se atuar diretamente, fomentando o processo inovativo, sem necessariamente ter a massa crítica de pesquisadores acadêmicos indispensáveis à geração do conhecimento na área".
\end{abstract}

\title{
3 Modelo dinâmico de inovação
}

Para Nikolsky (2010), deveríamos adotar um novo modelo de C\&T, denominado "modelo dinâmico de inovação", como o fizeram os principais países dos "Tigres Asiáticos" - Coréia, Taiwan e China, "que começaram a inovar e a crescer no cenário das patentes e da propriedade industrial bem antes de terem alguma significação na criação do conhecimento científico" (Nikolsky, 2010,p.37).

Enfim, o locus privilegiado das inovações tecnológicas é nas empresas e às universidades cabe o nobre papel da formação de recursos humanos altamente qualificados. Assim como, para o Estado, cabe-lhe a tarefa de regulamentar as ações estratégicas para o país, fomentar a inovação, compartilhar os riscos tecnológicos com os empresários e prover um crescimento sustentado de longo prazo e fiscalizar o correto uso do dinheiro público. Com essas políticas públicas, certamente, haverá mobilização das indústrias para a inovação. 
Economias desenvolvidas possuem sistemas nacionais de inovação articulados e consolidados e têm um padrão de interação entre ciência e tecnologia, que envolve: universidades/institutos de pesquisa e empresas - estabelecidas e novas. Ou seja, estão maduros em articular suas riquezas econômicas com as suas competências tecnológicas. Nesse contexto, os BRICS se inserem e a Índia é um bom exemplo, do que vem fazendo, ou seja, formando maciçamente mãode-obra qualificada para a indústria de softwares, que segundo Nikolsky (2010, p.38), "num curto espaço de tempo de doze anos, elevou em mais de 30 vezes as exportações com softwares".

As economias de industrialização recente, ou Tigres Asiáticos, ou "fenômeno Ásia" (Japão, Coréia, Taiwan, Hong-Kong, Cingapura, Indonésia, Malásia, Tailândia e Filipinas) também passaram por estágios ou transições de desenvolvimento econômico e tecnológico de 1960 a 1990. Notadamente, nos anos 1980, apresentaram grandes taxas de crescimento e rápida industrialização, ao adotarem medidas estratégicas de atração de investimentos externos, mão-deobra barata e disciplinada, isenção de impostos e baixos custos de instalação de empresas. No entanto, esse modelo econômico voltado para exportações tem sofrido críticas porque fica muito dependente dos países compradores de seus produtos, como por exemplo, os Estados Unidos, Japão e China. A Coréia do Sul é um exemplo desse modelo dinâmico de inovação, mas que apresenta ainda problemas em seu sistema nacional de inovação em transição (Kim, 2005). E a Malásia é outro exemplo de país aspirante a Tigre e à Sociedade do Conhecimento (Evers, 2001).

No Brasil, essa situação é particularmente importante, uma vez que os seus mestres e doutores, em sua grande maioria, estão trabalhando para as ICT e não, dentro dos laboratórios de P\&D das empresas inovadoras. Além do fato de que as empresas têm demandado cada vez mais a expertise das universidades e institutos de pesquisa pura ou aplicada, justamente por não contarem com esses profissionais altamente qualificados em suas bases, resultando com isso, uma baixa motivação para inovar, tornando-se um fator limitador da inovação industrial, conforme dados até 2008 da última PINTEC/IBGE, publicados em 2010.

O ex- Ministro do Ministério da Ciência, Tecnologia e Inovação - Aloizio Mercadante foi enfático ao afirmar que:

"A participação de empresas, inclusive de estatais, no número de pedidos de patentes é de somente $53 \%$, ao passo que no Japão e na Alemanha é de cerca de 90\%. Ademais, as empresas brasileiras investem, em média, apenas cerca de 0,51\% do PIB em ciência e tecnologia, enquanto que no Japão, por exemplo, as empresas gastam cerca de $2,7 \%$. Já a pesquisa aplicada, aquela que gera inovação produtiva, ainda é insuficiente, especialmente quando comparamos a performance do Brasil com as de outras nações emergentes importantes" (Bom, 2011,p.7). 
Daí que, o conhecimento é o novo fator de produção de uma nova ordem mundial denominada Economia do Conhecimento e que precisa ser vivenciada em sua plenitude por todos aqueles (nações, organizações e indivíduos) que precisam coletar, tratar, usar e disseminar os conhecimentos novos gerados e produzidos a partir do correto uso dos fluxos informacionais de comunicação e informação (TIC) e redes. Tal é o caso das empresas industriais, comerciais e de prestação de serviços, além dos NIT, também.

Entendemos que os NIT são de fundamental importância para o fortalecimento do Sistema Nacional de Inovações, a partir de sua criação pela Lei N. 10.973/04 (Brasil, 2004), pelo fato deles procurarem levar ao conhecimento da sociedade todos os conhecimentos gerados e produzidos nas universidades e institutos de pesquisa, estreitando-se assim, cada vez mais, os laços com o sistema produtivo, apesar deste "modelo linear" receber críticas dos teóricos da inovação, como já descrito anteriormente.

Evidenciamos até aqui, que o caminho percorrido pelas ICT nem sempre tem sido aquele sugerido pelos teóricos da Economia da Tecnologia. No entanto, isso não quer dizer que as ICT brasileiras ao criarem ou fortalecerem os seus NIT estejam fazendo algo impróprio para a sua comunidade ou país. Muito pelo contrário, estão tentando criar ou fortalecer uma cultura de proteção de seus ativos intangíveis, bem como de transferência de tecnologias junto à comunidade universitária ou dos institutos/centros de pesquisa e aos empresários externos às ICT.

Por isso termos adiantado na introdução deste artigo aquele pensamento de que estamos procurando problemas, pois já temos muitas soluções nos portfolios dos NIT brasileiros. Ou seja, estamos querendo dizer que as ICT estão produzindo uma série de patentes, por exemplo, mediante o estudo de seus pesquisadores, sem, no entanto, receberem problemas reais da sociedade/ mercado para solucionar. Com isso, o que ocorre é que os portfolios dos NIT estão aumentando a cada dia, sem as ICT terem a certeza de que tais patentes serão transferidas com sucesso às indústrias que precisam fabricar seus produtos de forma inovadora e com valor agregado.

Evidentemente que não estamos querendo dizer que os pesquisadores das ICTs principalmente não saibam o que estão fazendo... É claro, que sabem! $\mathrm{E}$, estão produzindo, via de regra, pesquisas puras ou descobertas científicas geradoras de invenções radicais que ampliarão a fronteira tecnológica (Nikolsky, 2010, p.34). O problema é que esse modelo linear leva em média de 10 a 30 anos para amadurecer e representa pequena fração da ordem de $1 \%$ das patentes americanas nos EUA, além dessas descobertas não se transformarem em tecnologias competitivas de novos produtos ou processos, se a economia não dispuser, previamente, de ampla estrutura de P\&D no setor produtivo, com capacidade de investimento de longo prazo (Nikolsky, 2010, p.35). 
Apesar desse cenário, os NIT brasileiros têm conseguido com alguma dificuldade transferir suas tecnologias ou know-how à sociedade/mercados. Eles têm feito isso, por intermédio de contratos de transferência de tecnologias, e com os apoios governamentais (CNPq, CAPES, MCT, FINEP, ABDI, FAP, INPI etc) e não governamentais, tais como o FORTEC - Fórum Nacional de Gestores de Tecnologia, Inovação e Transferência de Tecnologia (www.fortec.org.br) .

\section{Núcleos de Inovação Tecnológica (NIT) no Brasil}

A inovação, nos últimos anos, tem-se consolidado como um importante fator de competitividade para as empresas e para os países, principalmente, por ser o vetor da Sociedade do Conhecimento. O desenvolvimento científicotecnológico e as inovações tecnológicas são peças-chave para o crescimento da economia, da produtividade e qualidade de produtos e processos inovadores e da geração do trabalho e renda para os povos.

Países desenvolvidos há muito tempo já se despertaram para essa realidade do mundo globalizado e, por isso mesmo, já incorporaram a ciência, a tecnologia e a inovação em suas políticas de desenvolvimento. O Brasil despertou-se para isso nos anos 90 e cada vez mais tem intensificado seus esforços em unir as estratégias das políticas de Estado de Ciência \& Tecnologia com as estratégias da política industrial, até então, desvinculadas.

O marco regulatório brasileiro que deu o "start" na criação e proliferação de Núcleos de Inovação Tecnológica (NIT) nas Instituições Científicas e Tecnológicas (ICT) foi a publicação da Lei N. 10.973, de 02/12/2004, conhecida como Lei de Inovação e que foi regulamentada pelo Decreto N. 5.563, de 11/10/2005. Esta Lei, em seu Artigo 17, determina que: "A ICT deverá dispor de Núcleo de Inovação Tecnológica, próprio ou em associação com outras ICT, com a finalidade de gerir sua política de inovação" (Brasil, 2004).

Evidentemente, em muitas universidades e institutos de pesquisa brasileiros já existiam NIT, muitos deles com outras denominações do tipo: escritórios de transferência de tecnologia, agências de inovação, núcleos de propriedade intelectual, etc. E com a Universidade Federal do Paraná (UFPR) também não foi diferente. Esta Universidade já dispunha de núcleos dispersos em seus campi, que não tinham sido reunidos sob a denominação de um único NIT.

Em meados de maio de 2006, em evento da Rede de Tecnologia do Rio de Janeiro, foi criado pelos gestores de NIT brasileiros o Fórum Nacional dos Gestores de Tecnologia, de Inovação e Transferência de Tecnologia (FORTEC www.fortec.org.br), do qual esta autora foi um dos signatários. Dentre os vários objetivos para a sua criação estava a "disseminação da cultura de inovação, da propriedade intelectual e da transferência de tecnologia" e o "mapeamento e divulgação das atividades e indicadores das Instâncias Gestoras de Inovação (IGI)" (Torkomian, 2009, p.22). 
O panorama dos NIT brasileiros que apresentamos, provêm, portanto, deste importante fórum e foi levantado, à época, por Torkomian (2009, p.21-37). Em 2006, quando de sua criação, o FORTEC contava com 120 NIT, sendo que 78 (65\%), responderam ao seu levantamento. Hoje, o FORTEC já conta com aproximadamente 200 NIT filiados, inclusive o da UFPR.

Sinteticamente, os dados coletados foram os seguintes: 1) em termos de distribuição geográfica, a maior parte dos NIT está na Região Sudeste (35\%); em termos de vinculação às ICT, os NIT estão vinculados principalmente às ICT públicas federais (53\%); em termos de natureza da ICT os à qual o NIT está vinculado, a maior parte deles está nas universidades (74\%); em termos de vinculação interna nas ICT os NIT estão vinculadas às pró-reitorias de Pós-Graduação e Pesquisa (30,5\%); em termos de idade dos NIT, a pesquisa mostrou que 52,5\% deles foram criados a partir de 2005; quanto à formalização dos NIT na estrutura da ICT, verificou-se que a maioria (59\%) foi criado através de instrumentos formais do tipo portarias das reitorias, resoluções de conselhos superiores etc; também observaram que tais NIT também têm uma regulamentação interna formalizada para a propriedade intelectual (44\%); verificou-se que 51\% dos NIT possuem regulamentações para a distribuição de royalties; em termos de equipe dos NIT, a maioria ( $82 \%$ ) não dispõe de mais de dez pessoas; quanto às atividades dos NIT, a maioria deles (90\%) dedicamse ao atendimento, orientação e acompanhamento dos processos relacionados à propriedade intelectual; em termos de procedência dos recursos financeiros para os NIT, os mesmos vêm da FINEP e do CNPq e de 2004 a 2007 vêm tendo um aumento expressivo; com relação aos recursos gerados pelos próprios NIT, a situação ainda é incipiente, apesar do seu crescimento; dentre os maiores problemas enfrentados pelos NIT, o principal deles é a contratação e capacitação de pessoal (77\%); quanto aos depósitos de patentes de invenção, 67\% dos NIT não possui mais do que dez pedidos requeridos no INPI e a UNICAMP responde por $26 \%$ do total de depósitos da Região Sudeste (a que mais deposita); quanto ao volume de patentes licenciadas ainda é muito pequeno; por fim, em termos de proteção de modelos de utilidade, marcas, programas de computador e cultivares, os depósitos ainda são poucos, entre 1 a 10 por ano.

Da análise desses dados coletados, Torkomian (2009, p.37) concluiu que além do problema sério da falta de capacitação dos NIT brasileiros, há, ainda, questões de fundo anteriores a serem resolvidas segundo a autora, tais como: dotação orçamentária, recebimento de vagas, natureza das ICT, capacitação para os licenciamentos de tecnologias geradas nas ICT e as diferenças regionais que persistem no país.

No entanto, apesar dessas constatações, o FORTEC, segundo a autora (Torkomian, 2009, p.37), tem desempenhado um importante papel nesse contexto, por meio das seguintes ações: "capacitação dos NIT; ação junto ao Comitê de Avaliação da Lei de Inovação; ação conjunta com o MCT para manutenção de 
base de dados atualizada; disseminação das informações referentes às boas práticas de gestão e contribuição na proposição de políticas públicas que possam fortalecer os NIT e alavancar a geração da inovação que o país precisa".

Amadei e Torkomian (2009, p.10-18) analisaram os depósitos de patentes das universidades públicas paulistas no INPI, no período de 19952006, perfazendo 672 registros. Pesquisaram as seguintes universidades: UNICAMP, USP, UNESP, UFSCar e UFSP. Constataram um avanço, mesmo que instável, do número de depósitos de patentes dessas universidades, das quais a UNICAMP responde por $60 \%$ dos registros.

Essa realidade permanece em crescimento constante. De acordo com os dados apresentados sobre os NIT brasileiros, no Workshop Nacional dos Núcleos de Inovação Tecnológica, promovido pela Secretaria de Desenvolvimento Tecnológico e Inovação do MCT, com apoio do CGEE e do FORTEC, nos dias 8 e 9 de novembro de 2010, em Brasília/DF, do qual tivemos o privilégio de participar, houve um aumento considerável no número de NIT, que em 2006 era de 40 e em 2010, chegou a 161, revelando um crescimento aproximado de $50 \%$ ao ano. Com isso, vê-se que os NIT são "agentes fundamentais da articulação entre a academia e o setor produtivo, com a responsabilidade de propor, acompanhar e avaliar as políticas de inovação para promover a proteção e a manutenção da propriedade intelectual, e para transferir as novas tecnologias para o setor empresarial".

Esse evento contou com a adesão de 500 participantes, que trocaram experiências de "boas práticas", nas seguintes categorias de classificação:

-Ferramentas de gestão;

-Uso dos instrumentos e políticas públicas de incentivo à inovação;

-Transferência de tecnologia;

-Estruturação do NIT;

-Praticas de divulgação de tecnologias;

-Políticas internas.

Além dessa troca de experiências, os participantes também tiveram a oportunidade de refletir sobre as possibilidades de aprimoramento do Formulário para Informações sobre a Política de Propriedade Intelectual das Instituições Científicas e Tecnológicas do Brasil (FORMICT), criado pela SETEC/MCT para coleta de informações dos NIT brasileiros. 


\subsection{Núcleo de Inovação Tecnológica do Paraná (NITPAR) 4}

No que tange ao estado do Paraná, cumpre-nos salientar que, em meados de agosto de 2002, foi criada a Agência Paranaense de Propriedade Industrial (APPI), com apoio do Instituto de Tecnologia do Paraná (TECPAR) e da Fundação Araucária. Em dezembro de 2002, foi criada também, a Rede Paranaense de Gestão em Propriedade Intelectual, com apoio da FINEP/CNPq e coordenada pela APPI/TECPAR. E, em meados de 2007, foi criado o Núcleo de Inovação Tecnológica do Paraná (NITPAR), com apoio da Secretaria de Estado da Ciência, Tecnologia e Ensino Superior (SETI) e da FINEP. A relevância de todas essas iniciativas paranaenses, em especial, a do NITPAR, se explicam por meio de seus objetivos estratégicos, quais sejam: ampliar as cooperações em termos de inovação entre as empresas e as ICT estaduais, apoiar e facilitar a transferência de tecnologia das ICT paranaenses para o mercado e estimular e apoiar a inovação em empresas e pesquisas de base tecnológica. Além desses objetivos, o NITPAR conta com uma carteira de serviços dinâmica e atualizada, como transcrito a seguir:

-capacitação em propriedade intelectual;

-oficinas pró-inovação;

-monitoramento tecnológico;

-pesquisa de anterioridades;

-diagnóstico e promoção de capacidades para inovação;

-levantamento de ofertas e demandas tecnológicas para o agronegócio;

-estímulo ao empreendedorismo;

-estímulo à incubação de projetos inovadores.

A institucionalização do NITPAR foi feita com o apoio de vários parceiros, dentre eles, a UFPR e a UTFPR.

\subsection{Agência de Inovação UFPR}

Já se disse que a inovação é um conceito que sempre esteve presente na UFPR, desde 1912 - data de sua fundação. Entretanto, isso é correto do ponto de vista da qualidade da produção científica que é produzida na instituição. Porém, do ponto de vista da cultura de proteção da propriedade intelectual e da transferência de tecnologia, incluindo uma política de proteção de seus ativos intangíveis, não. Na verdade, isso data de meados do ano de 2003, quando a Pró- 
Reitoria de Pesquisa e Pós-Graduação (PRPPG) resolveu consultar a comunidade universitária para criar documento a ser discutido e aprovado no Conselho Universitário (COUN) sobre a regulamentação da propriedade intelectual no âmbito da UFPR. Isso foi feito e resultou na Resolução N. 09/2003 - COUN (UFPR,2003). Portanto, há vários meses antes da aprovação da Lei de Inovação. Em paralelo, abriram-se as portas do Núcleo de Propriedade Intelectual (NPI) na PRPPG, o qual perdurou por seis anos naquela Pró-Reitoria.

Antes, porém, em meados de 2001, no campus Centro Politécnico da UFPR, começava a funcionar o seu Núcleo de Empreendedorismo e Projetos Multidisciplinares (NEMPS), que tinha como missão fomentar o nascimento de empresas incubadas de base tecnológica.

Em 2005, foi a vez do surgimento do Portal de Relacionamento da UFPR. Este, por sua vez, abrigava o Escritório de Transferência de Tecnologia (ETT) da Universidade. Atendia a empresários e dirigentes de instituições governamentais e não-governamentais, na efetivação de parcerias para o desenvolvimento de produtos e processos de interesse ao desenvolvimento regional.

Dessa "dispersão geográfica" dentro da UFPR (cada núcleo funcionava num campus diferente) entre os atores ligados à gestão da inovação na instituição, acrescido o fato das equipes desses núcleos não interagirem com eficiência e eficácia, surgiu a idéia da criação de uma Agência de Inovação para a UFPR, para zelar pela política de inovação da instituição, bem como proceder às fiscalizações e demais regulamentações inerentes à área. Essa idéia ganhou força e adeptos e culminou com a aprovação da Resolução N. 16/2008 - COPLAD, que "Institui a Agência de Inovação Tecnológica da Universidade Federal do Paraná - AGITEC e seu Conselho Diretor e dá outras providências" (UFPR, 2008). Nessa época, a Pró-Reitora de Pesquisa e Pós-Graduação era a Profa. Dra. Maria Benigna Martinelli de Oliveira (in memoriam), que se dedicou com empenho e afinco na criação e aprovação da idéia de uma Agência de Inovação na UFPR.

Portanto, em termos de idade cronológica, a Agência de Inovação UFPR (seu nome atual registrado como marca no INPI) tem quase quatro anos, posto que fora aprovada em 28/05/2008. No entanto, em termos de atuação de NIT na instituição com apoio da Reitoria, isso data de 2001, quando da criação e funcionamento do NEMPS - seu núcleo de inovação mais antigo, antes do NPI e do ETT, portanto, é considerado um NIT bastante jovem e que não alcançou, ainda, a sua sustentabilidade, o que, em média, acontece nos EUA e na Europa, em torno dos 10 anos de existência.

O Quadro abaixo, ilustra o perfil da Agência de Inovação UFPR, conforme sua descrição na Resolução No 16/08-COPLAD: 
Quadro 1 - Perfil da Agência de Inovação UFPR

ELEMENTOS DESCRIÇÃO

Art. $1^{\circ}$-Contribuir para o desenvolvimento científico-tecnológico da

1 Finalidade sociedade brasileira, bem como seu desenvolvimento econômico e social.

Art. $1^{\circ} / 1^{\circ}$-Promover a articulação da universidade com a sociedade civil, aproximando a produção científica de suas aplicações práticas.

2 Missão

Através da colaboração entre academia, empresas e governos, a AGITEC buscará contribuir para o aumento da competitividade e relevância das pesquisas desenvolvidas na UFPR com o propósito da inovação.

Art. $1^{\circ} / \S 2^{\circ}$-Transformar radicalmente a relação entre a Academia e o Setor Produtivo, bem como a relação dos pesquisadores com o seu próprio trabalho, ao fomentar a cultura empreendedora e

3 Visão de proteção dos ativos intangíveis, bem como a transferência de tecnologia à sociedade, buscando ser uma referência para as IFES Instituições Federais de Ensino Superior - em matéria de integração Universidade/Empresa (U-E).

Art. $1^{\%} / \S 3^{\circ}$

I- Zelar pela política de inovação tecnológica da UFPR para estar em consonância com a

legislação em vigor;

II- Valorizar a pesquisa aplicada e que resulta em inovação tecnológica capaz de agregar valor econômico e melhoria da qualidade de vida da sociedade.

III- Articular parcerias estratégicas entre a UFPR e os setores

4 Objetivos empresariais, governamentais e não governamentais para atuar em projetos cooperativos de desenvolvimento científico tecnológico;

IV- Estimular o processo de pré-incubação e incubação de empresas inovadoras de base

tecnológica no âmbito da Universidade;

V- Difundir a cultura de proteção da propriedade intelectual

na Universidade para estimular o registro, o licenciamento e a comercialização dos ativos intangíveis;

VI- Disseminar a cultura empreendedora para toda a comunidade universitária.

\begin{tabular}{|c|c|}
\hline \multicolumn{2}{|c|}{ Quadro 1 (continuação) - Perfil da Agência de Inovação UFPR } \\
\hline 5 Justificativa & $\begin{array}{l}\text { Art. } 1^{\circ} / \S 4^{0} \text {-A justificativa para a criação da AGITEC na UFPR baseia- } \\
\text { se: } \\
\text { I- Na legislação brasileira que solicita a criação de NITs - Núcleos de } \\
\text { Inovação Tecnológica nas ICTs, para gerir a política de inovação em } \\
\text { articulação com a sociedade; } \\
\text { II- Na necessidade de transformar o conhecimento em valores } \\
\text { ecossocioambientais e econômicos; } \\
\text { III- Na necessidade de garantir a propriedade intelectual que pode } \\
\text { resultar em novas tecnologias; } \\
\text { IV- Na necessidade de criar mecanismos facilitadores entre } \\
\text { pesquisadores e a sociedade, visando a melhoria da qualidade de } \\
\text { ensino, pesquisa e extensão, bem como o desenvolvimento tecnológico } \\
\text { na Uniyersidade. }\end{array}$ \\
\hline $\begin{array}{l}6 \text { Estrutura } \\
\text { Organizacional }\end{array}$ & $\begin{array}{l}\text { Art.30-Orgão Suplementar, vinculado à Reitoria. } \\
\text { Possui um Conselho Diretor, uma Diretoria Executiva e } 3 \\
\text { Coordenaçoses, a saber: } \\
\text { I - Incubadoras de Empresas de Base Tecnológica; } \\
\text { II - Propriedade Intelectual; } \\
\text { III - Transferência de Tecnologia. } \\
\text { NITs nos campi e/ou setores da UFPR (a serem criados e gerenciados } \\
\text { de forma descentralizada e subordinados à Agência de Inovação). }\end{array}$ \\
\hline
\end{tabular}

Fonte: Resolução N ${ }^{\circ}$ 16/08-COPLAD/UFPR. 
É importante salientar que após a aprovação da Resolução $\mathrm{N}^{0}$ 16/08-COPLAD, em 28/05/2008, a estrutura organizacional da Agência de Inovação Tecnológica da UFPR - AGITEC (nome fantasia) ampliou-se com uma Secretaria Executiva e Assessoria Especial, conforme consta de seu regimento. Seu primeiro Diretor Executivo foi o Prof. Dr. Sérgio Scheer e esta autora, a sua primeira Coordenadora de Propriedade Intelectual.

\section{Considerações Finais}

Este artigo teceu algumas reflexões acerca da problemática da pesquisa científica no Brasil versus as inovações tecnológicas, sem pretender ser exaustivo. Ancorou-se, principalmente, no pensamento de Nikolski (2010), por corroborar com ele sobre os modelos de C\&T\&I.

Apesar das teorias, a prática social dos NIT tem sido positiva para o país. E, um dado interessante nesse sentido é o fato do registro das patentes terem crescido 64\% no país em dez anos (2001 a 2010), superando até mesmo, a Europa e o Japão (Garcia, 2012).

Com isso, evidencia-se que os NIT têm um papel fundamental no sistema brasileiro de inovações, principalmente, porque o maior número de registro de patentes, por exemplo, vem de empresas estatais e instituições públicas de pesquisa (Petrobras, UNICAMP, USP, FAPESP, UFMG) (GARCIA, 2012). Dois exemplos concretos e salutares mostrados neste artigo foram os NIT do estado do Paraná (NITPAR) e a Agência de Inovação UFPR, que detém um dos maiores portfolios de patentes da Região Sul do Brasil.

\section{Referências}

Amadei, J. R. P.; Torkomian, A. L. V. (2009) As patentes nas universidades: análise dos depósitos das universidades públicas paulistas (1995-2006). Ci. Inf., Brasília, vol.38, n.2, p.9-18.

Engenhar - o jornal da inovação (2011); Bom começo, São Paulo, p.7.

Brasil. Lei n. 10.973, de 2 de dezembro de 2004. Dispõe sobre incentivos à pesquisa científica e tecnológica no ambiente produtivo e dá outras providências. Disponível em: www. planalto.gov.br - Acesso em: 29/07/10.

Dausha, R. M. Definição de inovação em negócios para o Brasil. In: Parolin, S. R. H. ; Oliveira, H. C. de. (Org). Inovação e propriedade intelectual na indústria. Curitiba: SENAI/SESI, 2010. p.17-28.

Evers, H. D. Towards a Malaysian knowledge society. In: International Malaysian Studies Conference (MSC 3), III, Bangi. Proceedings... 6-8 Aug. 2001. 23p. Disponível em: Curso de Nivelamento ao EGC/UFSC 2011 - Unidade 1 - Acesso em: 09/08/11.

Garcia, R.; Patentes crescem 64\% no país em dez anos. Folha de São Paulo, caderno de ciências, p. C7, 06/03/12. 
Kim, L. (2005). O sistema nacional de inovação sul-coreano em transição. In: Kim, L. ; Nelson, R. R. (Org.). Tecnologia, aprendizado e inovação: as experiências das economias de industrialização recente. Campinas/SP: Ed. UNICAMP. p.449-483 (Coleção clássicos da inovação).

Lotufo, R. de A. (2009). A institucionalização dos núcleos de inovação tecnológica e a experiência da Inova UNICAMP. In: Santos, M. E. R. dos ; Toledo, P. T. M. de; Lotufo, R. de A. (Org.). Transferência de tecnologia: estratégias para a estruturação e gestão de núcleos de inovação tecnológica. Campinas/SP: Komedi. 350p. ; p.41-73.

Mattos, J. R. L. de ; Guimarães, L. dos S. (2005). Gestão da tecnologia e inovação: uma abordagem prática. São Paulo: Saraiva. 278p. ; p.5.

Nikolski, R. (2010). Modelo dinâmico para inovações tecnológicas. In: Parolin, S. R. H. ; Oliveira, H. C. de (Org.). Inovação e propriedade intelectual na indústria. Curitiba: SENAI/SESI. 151p. ; p.29-42. (Coleção inova, v.4).

Torkomian, A. L. V. (2009). Panorama dos núcleos de inovação tecnológica no Brasil. In: Santos, M. E. R. dos ; Toledo, P. T. M. de ; Lotufo, R. de A. (Org.). Transferência de tecnologia: estratégias para a estruturação e gestão de núcleos de inovação tecnológica. Campinas/SP: Komedi. 350p. ; p.21-37.

Universidade Federal do Paraná (UFPR). Secretaria de Órgãos Colegiados (SOC). Resolução n. 09 - COUN, de 2003. Dispõe sobre a regulamentação da propriedade intelectual no âmbito da UFPR e dá outras providências. Disponível em: www.ufpr.br/soc - Acesso em: 05/03/12.

Resolução n. 16 - COPLAD, de 28/05/08. Dispõe sobre a criação da AGITEC e dá outras providências. Disponível em: www.ufpr.br/soc -Acesso em: 05/03/12. 
